\title{
Study of a fluidized bed reactor for gasification of biomass
}

\author{
K. Kleiva, R. K. Thapa \& B. M. Halvorsen \\ Department of Process, Energy and Environmental Technology, \\ Telemark University College, Norway
}

\begin{abstract}
Experimental studies have been performed on a cold fluidized bed with glass and plastic particles emulating the properties of wood and olivine particles. The purpose of the experimental studies was to find the fluidization properties of the particles. These experimental studies have founded the background for conducting computational studies of multiphase flows of particles with the same properties. The computational studies are performed in ANSYS Fluent 12.0. The mathematical model has been verified with an experimental study to validate the simulations. A combination of steam, olivine particles and wood chips has then been simulated based on the results from the experimental and computational studies. The results from the computational studies show that the minimum fluidization velocity in the Fluent-simulations is higher than in the physical experiments, which is expected to a certain degree because of the wider distribution of particle sizes in the physical experiment. The pressures in the simulations scale well with the experimental results with increasing superficial velocity; more than one solid phase in the simulation gives a higher difference in pressure between the simulated and experimental values.

Keywords: fluidized bed, CFD, multi-phase Eulerian model, multiple density simulations, biomass, CHP.
\end{abstract}

\section{Introduction}

The behavior of biomass particles inside a fluidized bed is an essential part of the efficiency of a gasification combined heat and power-plant (CHP-plant), and for simulations of the behavior to be accurate, physical experiments are vital. The computational model used in fluent is based on already published models [1]. 
In order for a gasifying facility to be efficient and the gasifying temperatures to be adequately high, good mixing is necessary in the fluidized bed unit. The process itself should be able to generate enough heat so that external energy is not required to feed the process when it is up and running. A big span in size and density between the reacting particles and the inert particles in the bed lead to a natural segregation of the lighter and denser particles. The biomass tends to float on top of the bed, volatiles are released into the freeboard and the requirements for heating the steam inside the bed might be unfulfilled, making it difficult to maintain sufficient gasification temperature. This results in a much lower efficiency of the gasification process than what is possible with a more uniform mixing [2].

\section{Multiphase modeling in ANSYS fluent}

The adaptation of a mathematical model to a fluidized bed is already covered by Sanoja and Ariyarathna [3]. The setup used in Fluent is presented in Table 1.

Table 1: $\quad$ Mathematical model parameters in ANSYS fluent.

\begin{tabular}{|l|l|}
\hline Type of force & Mathematical model \\
\hline Solid-fluid drag model & Syamlal-O'Brien \\
\hline Solid-solid drag model & Syamlal-O'Brien Symmetric \\
\hline Granular viscosity & Syamlal-O'Brien \\
\hline Granular bulk viscosity & Constant \\
\hline Frictional viscosity & Schaeffer \\
\hline Frictional pressure & Based-ktgf \\
\hline Solids pressure & Ma-ahmadi \\
\hline Radial distribution function & Ma-ahmadi \\
\hline
\end{tabular}

The multiphase model used in the simulations is the Euler-Euler approach. The different mathematical models are presented in this section. Multiphase simulation solves the continuity, momentum and energy equations for each phase. For this study, the two most important are the continuity equation and the momentum equation as there is no transfer of heat between the phases. The minimum fluidization velocity $\left(\mathrm{u}_{\mathrm{mf}}\right)$ of a mixture characterizes its fluidization properties and is a way to compare the experiments with the simulations [4].

\subsection{Experimental setup for pressure drop measurements}

A small scale fluidized bed is used in the experimental study. The bed has pressure sensors located on the walls with a vertical distance of $10 \mathrm{~cm}$ between them. A drawing of the bed setup is shown in Figure 1. 


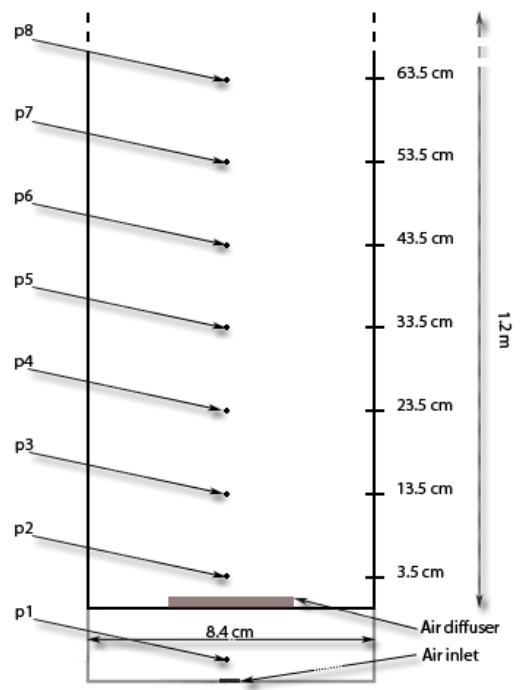

Figure 1: 2-D representation of the circular fluidized bed with dimensions and pressure sensor alignments (not drawn to scale).

The air diffuser, seen in Figure 1, in the bottom of the bed gives a uniform air distribution at the inlet of the bed. The eight pressure nodes are connected to a hub which again is connected to LabVIEW. The diameter of the circular bed is $8.4 \mathrm{~cm}$ and the height is $1.2 \mathrm{~m}$.

\subsection{Fluidized bed experiments}

Two types of particles were used in the experiments; their respective characteristics as well as the air phase characteristics used in the Fluent simulations are displayed in Table 2.

Table 2: Phase characteristics for the G, GP-1 and GP-2 simulations.

\begin{tabular}{|c|c|c|c|c|}
\hline Phase & $\begin{array}{c}\text { Mean } \\
\text { particle size } \\
(\boldsymbol{\mu m})\end{array}$ & $\begin{array}{c}\text { Density } \\
\left(\mathbf{k g} / \mathbf{m}^{\mathbf{3}}\right)\end{array}$ & $\begin{array}{c}\text { Viscosity } \\
(\mathbf{k g} / \mathbf{m}-\mathbf{s})\end{array}$ & $\begin{array}{c}\text { Geldart } \\
\text { classification [5] }\end{array}$ \\
\hline Glass & 182 & 2485 & - & $\mathrm{B}$ \\
\hline Plastic & 3000 & 964 & - & $\mathrm{D}$ \\
\hline Air & - & 1.225 & $1.7894 \mathrm{e}-05$ & - \\
\hline
\end{tabular}

Three sets of experiments were run with different mixtures of glass and plastic particles, as seen in Table 3 . 
Table 3: Mixture ratios of glass and plastic in the G, GP-1 and GP-2 simulations.

\begin{tabular}{|c|c|c|c|c|}
\hline Simulation & $\begin{array}{c}\text { Volume of } \\
\text { glass } \\
\text { particles (l) }\end{array}$ & $\begin{array}{c}\text { Volume of } \\
\text { plastic } \\
\text { particles (l) }\end{array}$ & Total mixture & $\begin{array}{c}\text { Weight } \\
\text { of plastic } \\
\text { particles }\end{array}$ \\
\hline G & 2 & 0 & $0 \mathrm{vol} \%$ plastic & $0 \mathrm{wt} \%$ \\
\hline GP-1 & 2 & 0.6 & $23 \mathrm{vol} \%$ plastic & $10.5 \mathrm{wt} \%$ \\
\hline GP-2 & 2 & 1 & $33 \mathrm{vol} \%$ plastic & $17.5 \mathrm{wt} \%$ \\
\hline
\end{tabular}

To transfer the experimental studies and simulation to a relevant case with material properties that are of the same kind that appear in a real situation, a steam/olivine/charred wood simulation has been performed. The characteristics of the different elements of this simulation are presented in Table 4.

Table 4: Phase characteristics for the steam/olivine/charred wood simulation.

\begin{tabular}{|c|c|c|c|c|}
\hline Phase & $\begin{array}{c}\text { Mean } \\
\text { particle size } \\
(\boldsymbol{\mu m})\end{array}$ & $\begin{array}{c}\text { Density } \\
\mathbf{( k g / \mathbf { m } ^ { 3 } )}\end{array}$ & $\begin{array}{c}\text { Viscosity } \\
\mathbf{( k g / m - s )}\end{array}$ & $\begin{array}{c}\text { Geldart } \\
\text { classification }\end{array}$ \\
\hline Steam $\left(800^{\circ} \mathrm{C}\right)$ & - & 0.29 & $4.1 \mathrm{e}-05$ & - \\
\hline Olivine & 400 & 2500 & $1.7894 \mathrm{e}-05$ & $\mathrm{~B}$ \\
\hline Charred wood & 3000 & 400 & $1.7894 \mathrm{e}-05$ & $\mathrm{D}$ \\
\hline
\end{tabular}

The mixture ratio of the olivine-charred wood mixture is displayed in Table 5. The weight-percentage of the charred wood particles is set to $10 \mathrm{wt}-\%$, giving a vol- $\%$ of 38.5 of the wood particles patched uniformly with olivines in the initial conditions in the fluidized bed simulation.

Table 5: $\quad$ Mixture ratios of olivine and charred wood in the simulation.

\begin{tabular}{|c|c|c|c|}
\hline $\begin{array}{c}\text { Volume of } \\
\text { olivine (l) }\end{array}$ & $\begin{array}{c}\text { Volume of charred } \\
\text { wood (cw) (l) }\end{array}$ & Total mixture & $\begin{array}{c}\text { Weight of cw- } \\
\text { particles }\end{array}$ \\
\hline 2 & 1.25 & $38.5 \mathrm{vol} \% \mathrm{cw}$ & $10 \mathrm{wt} \% \mathrm{cw}$ \\
\hline
\end{tabular}

\subsection{Results and discussion}

The snapshots from the simulations of the GP-1 mixture, Figure 2, show that small bubbles appear in the bed at around $8 \mathrm{~cm} / \mathrm{s}$, showing that $\mathrm{u}_{\mathrm{mf}}$ is reached. 

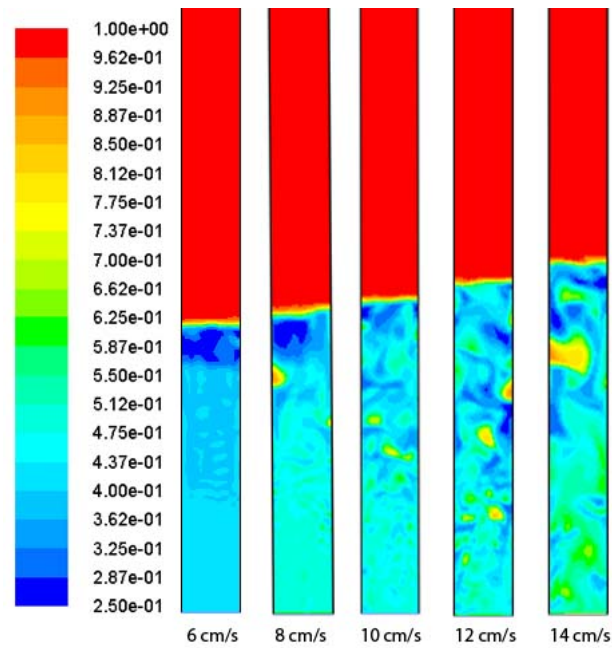

Figure 2: $\quad$ Volume fraction of air in a simulation of the GP-1 mixture.

The course of the volume fraction of air as a function of time in point $\mathrm{p} 3$, $13.5 \mathrm{~cm}$ above the air-inlet, of the GP-1 mixture for a superficial velocity of 6 and $8 \mathrm{~cm} / \mathrm{s}$ are shown in Figure 3 and Figure 4 respectively. Fluctuations in the volume fraction of air are a sign of fluidization.

Figure 3 and Figure 4 indicate, as the snapshot in Figure 2 that the $u_{m f}$ is reached before a superficial velocity of $8 \mathrm{~cm} / \mathrm{s}$.

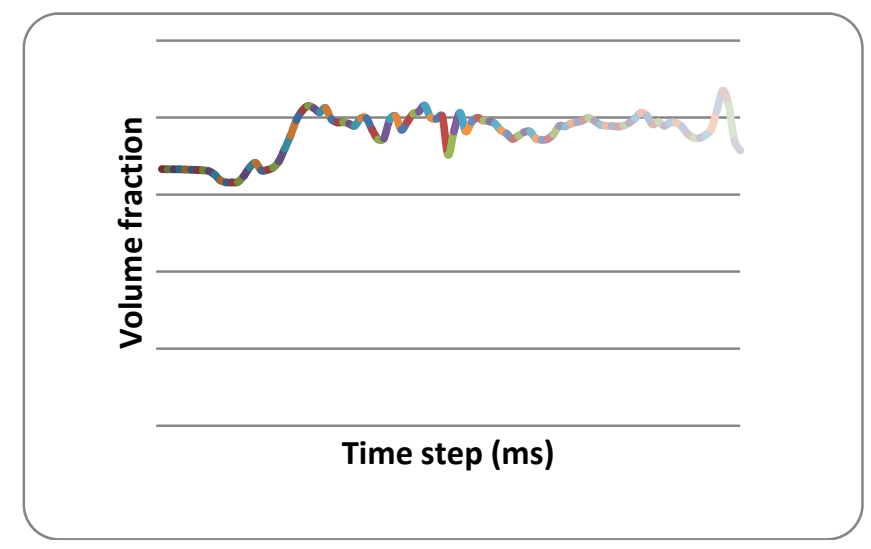

Figure 3: Course of the facet average volume fraction of air in p3 of the bed for the simulation of the GP-1 mixture at a superficial velocity of $6 \mathrm{~cm} / \mathrm{s}$ as a function of time. 


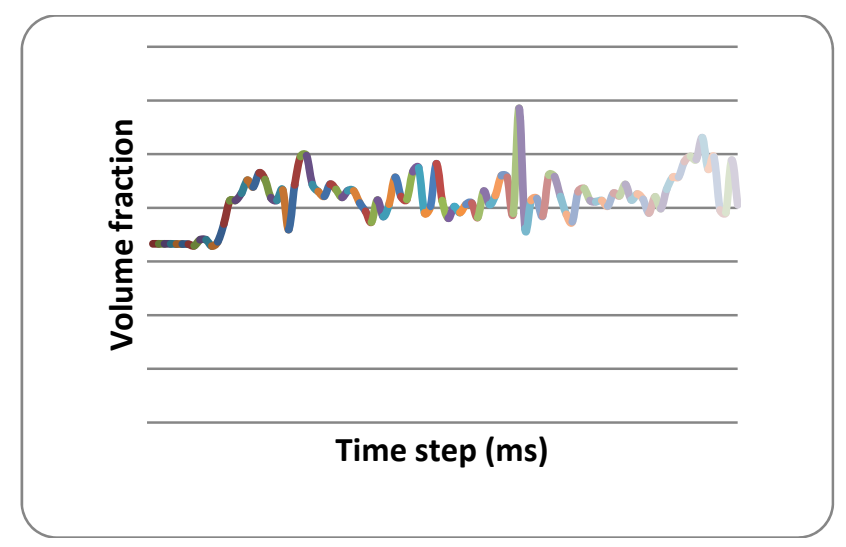

Figure 4: $\quad$ Course of the facet average volume fraction of air in $\mathrm{p} 3$ of the bed for the simulation of the GP-1 mixture at a superficial velocity of 8 $\mathrm{cm} / \mathrm{s}$ as a function of time.

The difference in the particle properties of the glass and plastic particles is clearly seen in Figure 5. The plastic particles float to the top because of their lower density and larger diameter, as also was visually apparent in the physical experiment. The snapshot at $14 \mathrm{~cm} / \mathrm{s}$ has a more uniform mixture of glass and plastic particles, suggesting that the vertical mixing of the two particle types is better with increased superficial velocity from $u_{\mathrm{mf}}$. The expansion of the bed is also making the distribution of plastic particles more uniform.

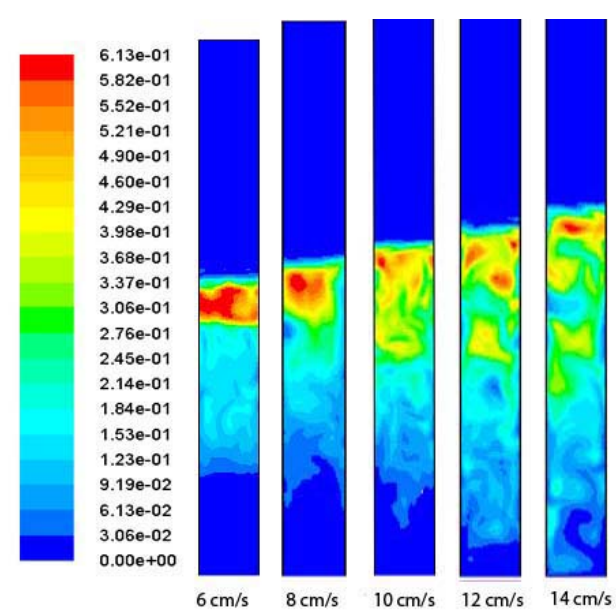

Figure 5: Volume fraction of plastic particles in a simulation of the GP-1 mixture.

The GP-2 mixture, Figure 6, has a slightly lower $\mathrm{u}_{\mathrm{mf}}$ than the GP-1 mixture, and this picture also shows bubble appearance at $6 \mathrm{~cm} / \mathrm{s}$ in the top part of the 


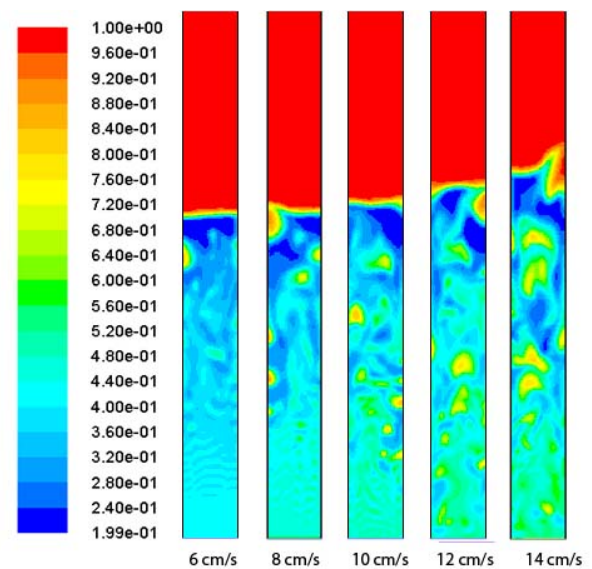

Figure 6: Volume fraction of air in a simulation of the GP-2 mixture.

bed. This holds true until enough light particles are added to the mixture to reduce the $u_{\mathrm{mf}}$ once again when the packing no longer is optimal.

Ramakers et al. [6] stated that a mixture of $10 \mathrm{wt}-\%$ of wood particles in a olivine-wood mixture is an optimal mixture for uniform mixing of the particles in a fluidized bed. This theory is arguably notable in the comparison of Figure 5 and Figure 7 which have a wt-\% of 10.5 and 17.5 respectively. The gathering of plastic particles in the top part of the bed is much higher in the GP-2 mixture in Figure 7 at a superficial velocity of $14 \mathrm{~cm} / \mathrm{s}$, which in a gasifier would result in a

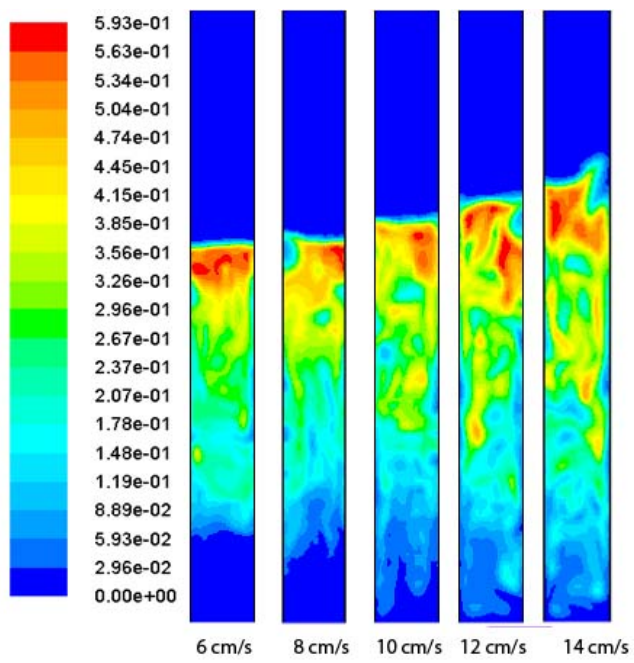

Figure 7: Volume fraction of plastic particles in a simulation of the GP-2 mixture. 
poorer energy transfer from the non-reacting olivine particles to the wood particles. Both particle mixtures have a uniform concentration in the bottom part of the bed, but the GP-1 mixture have a more uniform concentration overall.

\section{Particle mixing in a steam/olivine bed}

The simulation of the steam/olivine/charred wood-combination has been done to transfer the proven model to a case that is closer to a real gasifier in a CHP-plant.

A simplified theoretical value of the $\mathrm{u}_{\mathrm{mf}}$ for this mixture has been calculated using the Ergun equation [4] to be approximately $0.48 \mathrm{~m} / \mathrm{s}$. The course of the volume fraction of steam in the lowest superficial velocity simulation of 0.24 $\mathrm{m} / \mathrm{s}$, Figure 8 , shows that the $\mathrm{u}_{\mathrm{mf}}$ already is superceded. The large peaks in the volume fraction of steam are an indication of bubbles and the amplitude of the peaks are a sign of a well fluidized bed.

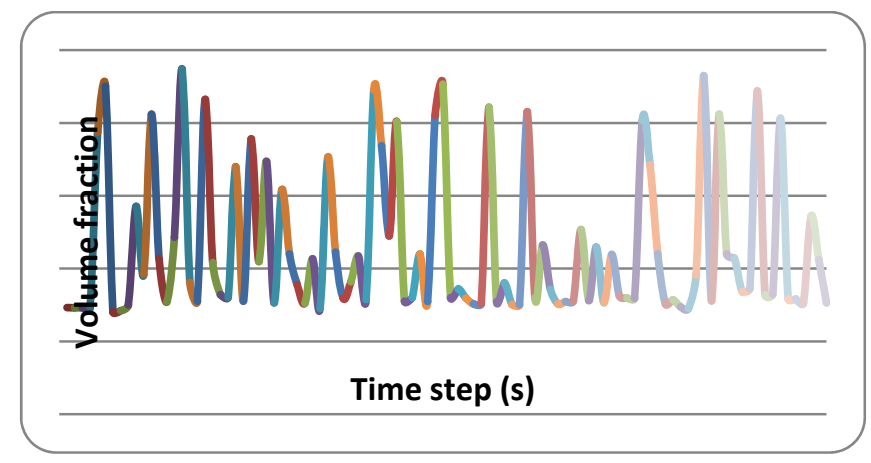

Figure 8: Volume fraction of steam in the steam/olivine/charred wood simulation, with a superficial velocity of $0.24 \mathrm{~m} / \mathrm{s}$ as a function of time.

When steam is used as a fluidizing medium, the superficial velocity required to achieve fluidization is increased when compared to cold air as a fluidization medium. This is because of the lower density of the steam. The lowest simulated velocity of $0.24 \mathrm{~m} / \mathrm{s}$ gives a proper fluidized bed, as can also be seen in Figure 9 . The bubbles are comparable to the GP-1 simulation, but it is apparent that the superficial velocity of $0.24 \mathrm{~m} / \mathrm{s}$ is slightly higher than the $\mathrm{u}_{\mathrm{mf}}$ for the particle-gas mixture. The most homogenous mixing of the combination of olivine and charred wood particles, see Figure 10, seems to be somewhere in between a superficial velocity $0.48 \mathrm{~m} / \mathrm{s}$ and $0.72 \mathrm{~m} / \mathrm{s}$. This can be stated because the area on the top the bed with charred wood at $0.72 \mathrm{~m} / \mathrm{s}$ has a higher concentration of wood particles than the top part at $0.48 \mathrm{~m} / \mathrm{s}$. This also supports that a higher superficial velocity to a certain points increases the buoyancy forces working on the lighter particles in the fluidized bed. The assumed $\mathrm{u}_{\mathrm{mf}}$ of slightly below 0.24 $\mathrm{m} / \mathrm{s}$ along with the fact that the most uniform mixing happens in between 0.48 
$\mathrm{m} / \mathrm{s}$ and $0.72 \mathrm{~m} / \mathrm{s}$ further supports the suggestion by Ramakers et al. [6], that a superficial velocity of 3-4 times the $u_{m f}$ is the ideal superficial velocity for optimal mixing of wood particles and non-reactive particles in a fluidized bed.

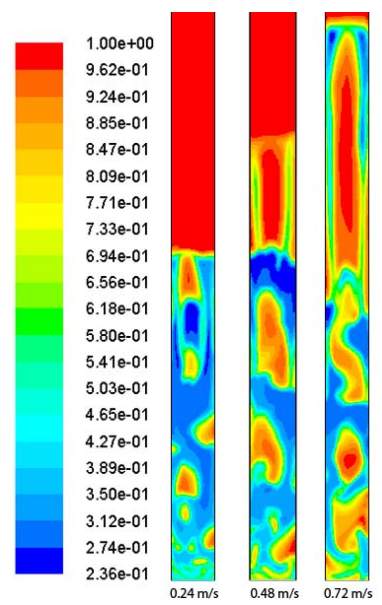

Figure 9: Volume fraction of steam in the fluidized bed.
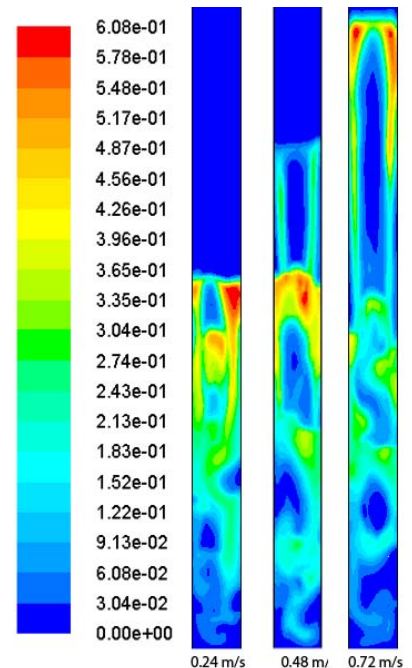

Figure 10: Volume fraction of charred wood in the fluidized bed.

\section{Conclusion}

The glass and plastic experiments show that adding plastic particles to the mixture causes an increase of the minimum fluidization velocity $\left(\mathrm{u}_{\mathrm{mf}}\right)$ to a certain 
point, before it decreases again. The addition of larger particles causes the packing factor of the glass particle mixture to increase to a degree, and hence the $\mathrm{u}_{\mathrm{mf}}$ to increase. When more light density and large particles are added to the mix, the $\mathrm{u}_{\mathrm{mf}}$ will start decreasing again as they have a smaller theoretical $\mathrm{u}_{\mathrm{mf}}$, the mixture of 23 vol- $\%$ plastic was found to have a $u_{m f}$ of $0.05 \mathrm{~m} / \mathrm{s}$, while the 33 vol- $\%$ plastic mixture was found to have a $u_{m f}$ of $0.045 \mathrm{~m} / \mathrm{s}$. In the case of mixed glass and plastic particles, the fluidization seemed to appear in both the top and bottom region of the fluidized bed simultaneously.

The simulations gave a higher $\mathrm{u}_{\mathrm{mf}}$ than the experiments for both mixtures of glass and plastic particles as well as for glass particles only. This is an effect of not having a particle distribution in the particles, only a mean diameter. The 23 vol- $\%$ plastic mixture has a simulated $u_{m f}$ of $0.09 \mathrm{~m} / \mathrm{s}$, while a simulated 33 vol- $\%$ plastic mixture has a $\mathrm{u}_{\mathrm{mf}}$ of $0.075 \mathrm{~m} / \mathrm{s}$.

The 23 vol\% plastic mixture correlates to a 10.5 mass $\%$ mixture, which is assumed to be the optimum mixture between large light density particles and small high density particles. This mixture gives a more uniform distribution of reacting and catalytic particles in the bed. When a 10 mass $\%$ mixture of wood chips in a steam, olivine and charred wood mixture is simulated, it is apparent that the uniformity of the mixture does not get better with a large superficial velocity. Large superficial velocities increase the buoyancy effect, causing the light particles to gather in the top of the particle region in the bed. A superficial velocity of 3-4 times the $u_{m f}$ seems to be the optimal conditions for a steam/olivine/wood chip gasifier.

\section{References}

[1] P. L. N. Ramesh and M. Raajenthiren, “A Review of Some Existing Drag Models Describing the Interaction Between the Solid-Gaseous Phases In a CFB," International Journal of Engineering Science and Technology, vol. 2, pp. 1047-1051, 2010.

[2] J. X. Laihong Shen, Fredrik Niklasson, Filip Johnsson, "Biomass mixing in a fluidized bed biomass gasifier for hydrogen production," Chemical Engineering Science, pp. 636-643, 2006.

[3] D. G. A. Sanoja U. Ariyarathna, "Recommendation of a Model for Simulating \& Analysis of the Influence of Particle Size Distribution on the Simulations of Bubbling Fluidized Beds," Master Thesis, Telemark College University, 2008.

[4] D. Kunii and O. Levenspiel, Fluidization Engineering: ButterworthHeinemann, 1991.

[5] D. Geldart, "Types of Gas Fluidization," Powder Technology, pp. 285-295, 1973.

[6] Bram J. Ramakers, Ronny de Ridder and Piet J.A.M. Kerkhof "Fluidization behavior of wood/sand mixtures," Drying, pp. 1337-1344, 2004. 